\title{
Prognostic Significance of CD109 Expression in Patients with Ovarian Epithelial Cancer
}

So Young Kim · Kyung Un Choi ${ }^{1}$ Chungsu Hwang · Hyung Jung Lee Jung Hee Lee · Dong Hoon Shin Jee Yeon Kim · Mee Young Sol Jae Ho Kim ${ }^{2} \cdot \mathrm{Ki}$ Hyung $\mathrm{Kim}^{3}$ Dong Soo Suh ${ }^{3} \cdot$ Byung Su Kwon ${ }^{3}$

Department of Pathology, Pusan National University Yangsan Hospital, Yangsan; ${ }^{1}$ Department of Pathology, Pusan National University Hospital, Busan; ²Department of Physiology, Pusan National University School of Medicine, Busan; ${ }^{3}$ Department of Obstetrics and Gynecology, Pusan National University Hospital, Busan, Korea

Received: December 26, 2018

Revised: March 17, 2019

Accepted: April 16, 2019

\section{Corresponding Author}

Kyung Un Choi, MD, PhD

Department of Pathology, Pusan National University

Hospital, Busan National University School of

Medicine, 179 Gudeok-ro, Seo-gu, Busan 49241 ,

Korea

Tel: +82-51-510-8055

Fax: +82-51-510-8140

E-mail: kuchoi@pusan.ac.kr
Background: Ovarian epithelial cancer (OEC) is the second-most common gynecologic malignancy. CD109 expression is elevated in human tumor cell lines and carcinomas. A previous study showed that CD109 expression is elevated in human tumor cell lines and CD109 plays a role in cancer progression. Therefore, this study aimed to determine whether CD109 is expressed in OEC and can be useful in predicting the prognosis. Methods: Immunohistochemical staining for CD109 and reverse transcription-quantitative polymerase chain reaction was performed. Then we compared CD109 expression and chemoresistance, overall survival, and recurrence-free survival of OEC patients. Chemoresistance was evaluated by dividing into good-response group and poor-response group by the time to recurrence after chemotherapy. Results: CD109 expression was associated with overall survival $(p=.020)$, but not recurrence-free survival $(p=.290)$. CD109 expression was not an independent risk factor for overall survival due to its reliability (hazard ratio, 1.58; $p=.160 ; 95 \%$ confidence interval, 0.82 to 3.05), although we found that CD109 positivity was related to chemoresistance. The poor-response group showed higher rates of CD109 expression than the good-response group (93.8\% vs $66.7 \%, p=.047$ ). Also, the CD109 mRNA expression level was 2.88 times higher in the poor-response group as compared to the good-response group $(p=.001)$. Conclusions: Examining the CD109 expression in patients with OEC may be helpful in predicting survival and chemotherapeutic effect.

Key Words: Ovarian epithelial carcinoma; CD109; Prognosis
Ovarian epithelial cancer (OEC) is the second most common gynecologic malignancy. ${ }^{1}$ However, it is often found in advanced stages, which makes it difficult to treat. OEC is believed to have an insidious onset, with no early symptoms. ${ }^{2}$ Survival rates for women with advanced disease range from $20 \%-30 \%$, which are much lower than those for women with early stage disease $(70 \%-$ $90 \%) .{ }^{3}$ Despite tumor-debulking surgery and chemotherapy, the 50-year survival rate is as low as $40 \% .{ }^{4}$ Although factors associated with the prognosis of OEC, such as p53 and human epidermal growth factor receptor 2 expression, have been reported, ${ }^{5,6}$ only a few factors can predict poor outcome in patients with OEC.

CD109 is a glycosylphosphatidylinositol-linked cell surface glycoprotein and a member of the 2-macroglobulin-C3, C4, C5 family of thioester-containing proteins. ${ }^{7} \mathrm{CD} 109$ expression is limited in certain cell types in normal tissues, including myoepithelial cells of the breast, salivary gland, and basal cells of the prostate. ${ }^{8}$ CD109 protein is a component of the transforming growth factor $\beta 1$ (TGF- $\beta 1$ ) receptor system, which is involved in cell proliferation and differentiation and has both tumor-suppressive and promoting effects during carcinogenesis. ${ }^{9}$ However, its functions are still unknown. A previous study showed that CD109 expression is elevated in human tumor cell lines and CD109 plays a role in cancer progression. ${ }^{10} \mathrm{CD} 109$ expression is significantly increased in carcinomas of the lung, gallbladder, uterine cervix, and vulva and soft tissue sarcomas. ${ }^{10-14}$ In addition, CD109 expression in lung squamous cell carcinoma is associated with the tumor stage, and its expression in myxofibrosarcomas is useful to predict recurrence. ${ }^{11,15}$ Thus, CD109 could be expressed in OEC and 
may be a useful predictor of OEC prognosis.

The aim of this study was to evaluate CD109 expression by immunohistochemistry and reverse transcription-quantitative polymerase chain reaction (RT-qPCR) using clinical specimens of human OEC; to analyze the correlation between CD109 expression and survival rate, recurrence rate, and chemotherapy response in patients with $\mathrm{OEC}$; and to investigate the prognostic significance of CD109 expression.

\section{MATERIALS AND METHODS}

\section{Patients and data}

OEC patients who underwent surgical resection at Pusan National University Hospital from 1998 to 2009 were selected for this study. All patients provided written informed consents and underwent surgical procedures. The biospecimens and data used for this study were provided by the Biobank of Pusan National University Hospital (PNUH), a member of the Korea Biobank Network. All samples derived from the National Biobank of Korea were obtained with the approval of institutional review board. After exclusion of cases with insufficient tissue material and clinical information, a total of 120 cases were enrolled and representative formalin-fixed paraffin tissue blocks were collected. Pathological data including histological type, pathological stage, tumor histological grade, nuclear grade, and mitosis were obtained from the primary pathology report. The histological tumor type was classified according to the World Health Organization criteria. ${ }^{16}$ Histological grades were classified as well-, moderately, and poorly differentiated according to the Silverberg grading system. ${ }^{17}$ The tumors were staged according to the International Federation of Gynecology and Obstetrics (FIGO) staging system (Table 1$){ }^{18}$

Fresh tissue samples of OEC were obtained by surgical resection and stored in the Biobank of PNUH. For this study, tissue samples from 12 patients who underwent resection were examined. We evaluated 12 patients with serous carcinoma who received chemotherapy. Six patients who did not experience relapse for 18 months after chemotherapy were included in the goodresponse group, and six patients who experienced recurrence within 6 months of chemotherapy initiation to 8 months of chemotherapy completion were included in the poor-response group (Table 2).

\section{Immunohistochemistry}

CD109 expression was assessed using CD109 immunohistochemical staining. The slides were then dewaxed in xylene and
Table 1. Characteristic of the patients

\begin{tabular}{lc}
\hline & No. $(\%)(n=120)$ \\
\hline Follow-up, median (range, mo) & $50(1-115)$ \\
Age at diagnosis (yr) & $51(15-82)$ \\
$\quad<50$ & $57(47.5)$ \\
$\geq 50$ & $63(52.5)$ \\
Histologic type & \\
Serous & $59(49.2)$ \\
Mucinous & $22(18.3)$ \\
Endometrioid & $9(7.5)$ \\
Clear cell & $27(22.5)$ \\
Undifferentiated & $3(2.5)$ \\
Histologic grade & \\
Well differentiated & $28(23.3)$ \\
Moderate differentiated & $61(50.8)$ \\
Poorly differentiated & $31(25.8)$ \\
Nuclear grade & \\
I & $6(5.0)$ \\
II & $65(54.2)$ \\
III & $49(40.8)$ \\
FIGO stage & \\
I & \\
II & $51(42.5)$ \\
III & $6(5)$ \\
IV & $44(36.7)$ \\
Mitosis (per 10HPFs) & $19(15.8)$ \\
1-9 & \\
$\geq 20-19$ & $45(37.5)$ \\
Overall survival & $41(34.2)$ \\
Survival & $34(28.3)$ \\
Death & \\
Absent & $63(52.5)$ \\
\hline
\end{tabular}

FIGO, International Federation of Gynecology and Obstetrics; HPF, highpower field.

dehydrated in ethanol. Staining was performed using BondMaxautostainer and other reagents (Leica Microsystems, Berlin, Germany). Deparaffinization was performed automatically in the autostainer with BondWash solution (Leica Microsystems) at $72^{\circ} \mathrm{C}$ for 30 minutes. After washing, the slides were incubated overnight at $4^{\circ} \mathrm{C}$ with a rabbit polyclonal anti-CD109 antibody (dilution 1:50, cat. No., HPA009292, Sigma-Aldrich, Merck KGaA, Darmstadt, Germany) and incubated with a horseradish peroxidase-conjugated goat anti-rabbit secondary antibody (dilution 1:100, cat. No., SA00001-2, ProteinTech Group, Inc., Wuhan Sanying Biotechnology, Wuhan, China) for 90 minutes at room temperature. Antibody binding was performed by incubating the slides for 1 minute with a solution of 1 drop of 3,3'-diamino-benzidine $(20 \times)$ per $1.0 \mathrm{~mL}$ diamino-benzidine substrate buffer (cat. No. ZLI-9017, Origene Technologies, Inc., 
Table 2. Fresh tissue samples of ovarian epithelial carcinoma

\begin{tabular}{lccccc}
\hline Case No. & Histologic type & Stage & Chemotherapy regimen & Period before recurrence (mo) & Response \\
\hline 1 & Serous & Illc & Carbo-Taxol & 4 & Poor response \\
2 & Serous & IV & Carbo-Taxol & 8 & Poor response \\
3 & Serous & IIIc & Carbo-Taxol & 4 & Poor response \\
4 & Serous & IIIc & Carbo-Taxol & 0 & Poor response \\
5 & Serous & IV & Carbo-Taxol & 1 & Poor response \\
6 & Serous & IV & Carbo-Taxol & 0 & Poor response \\
7 & Serous & IIlc & Carbo-Taxol & No recur & Good response \\
8 & Serous & IIlc & Carbo-Taxol & No recur & Good response \\
9 & Serous & IIlc & Carbo-Taxol & No recur & Good response \\
10 & Serous & Ila & Carbo-Taxol & 38 & Good response \\
11 & Serous & IIlc & Carbo-Taxol & No recur & Good response \\
12 & Serous & IV & Carbo-Taxol & 25 & Good response \\
\hline
\end{tabular}

apacilitaxel and carboplatin.

Beijing, China). The slides were then counterstained with EnVision FLEX hematoxylin (Dako, Agilent Technologies, Inc., Santa Clara, CA, USA) for 1 minute and dehydrated using ethanol and xylene. For negative control, staining was performed without primary antibody.

\section{Assessment of immunohistochemical staining}

Slides were evaluated using light microscopy. CD109 expression was detected through cytoplasmic and/or membranous staining of the tumor cells. The CD109 positivity of tumor cells was determined as follows: positive staining, $\geq 10 \%$ positive tumor cells and negative staining, $<10 \%$ positive tumor cells.

\section{RT-qPCR}

The mRNA levels of CD109 in the fresh tissue samples of OEC were measured using RT-qPCR. Total RNA was purified from cells using RNeasy mini prep kits (Qiagen, Valencia, CA, USA). cDNA was synthesized from $1 \mu \mathrm{g}$ RNA using the ProtoScript First Strand cDNA Synthesis Kit (New England Biolabs, Ipswich, MA, USA). Differential RNA levels were assessed using Luna Universal qPCR Master Mix (New England Biolabs) and primers for each gene. Quantitative PCR reactions were performed using an ECO Real-Time PCR system (PCRmax, Straffordshire, UK) as follows: $95^{\circ} \mathrm{C}$ for 30 minutes followed by 40 cycles at $95^{\circ} \mathrm{C}$ for 10 seconds, $61^{\circ} \mathrm{C}$ for 30 seconds, and $72^{\circ} \mathrm{C}$ for 20 seconds. The PCR products were analyzed using ECO ware (PCRmax). All samples were normalized to the signal generated from glyceraldehyde 3-phosphate dehydrogenase using the primers presented in Table 3. The CD109 gene expression was generalized by comparing the relative expression of $\mathrm{CD} 109$ with the internal standard glyceraldehyde 3-phosphate dehydrogenase using the $2^{-\Delta \Delta C T}$ method. ${ }^{19}$
Table 3. Primer sequences

\begin{tabular}{ll}
\hline Gene & \multicolumn{1}{c}{ Sequence (5'-3') } \\
\hline Cluster of differentiation 109 & \\
Forward & GAAGCCATCTCTCAACTTCACA \\
Reverse & CTCCTTGGAGGCCATGTG \\
GAPDH & \\
Forward & GAAGGTGGTGAAGCAGGC \\
Reverse & TTCCACTGTTAGATCCGCTCC \\
\hline
\end{tabular}

GAPDH, glyceraldehyde 3-phosphate dehydrogenase.

\section{Statistical analysis}

The Pearson's chi-square test was used to assess statistical significance between CD109 expression and various clinicopathological characteristics. Overall survival and recurrence-free survival were estimated using Kaplan-Meier plots. Multivariate analyses to determine hazard ratios for overall survival and recurrence-free survival were performed using Cox regression analysis. All analyses were performed using SPSS software (IBM, Armonk, NY, USA). A p-value of $<0.05$ was considered statistically significant.

\section{RESULTS}

\section{Patient characteristics}

A series of 120 OEC cases was retrieved for this study. The follow-up period ranged from 1 to 115 months (median, 50 months), and the patient age ranged from 15 to 82 years (median, 50 years). There were 59 cases of serous carcinoma, 22 cases of mucinous carcinoma, nine cases of endometrioid carcinoma, 27 cases of clear cell carcinoma, and three cases of undifferentiated carcinoma. The overall survival rate was $52.5 \%$, and the recurrence rate was $77.5 \%$. Other clinicopathologic parameters are presented in Table 1. 


\section{CD109 expression in OEC}

In OEC samples, the malignant tumor cells exhibited brownish CD109 staining in the membrane and cytoplasm (Fig. 1). CD109 expression was detected in 63 of the 120 OEC samples (52.5\%). CD109 expression differed according to the histological type of tumor $(\mathrm{p}<.001)$. There was a statistically significantly increase in the expression of CD109 in serous, endometrioid, and undifferentiated carcinomas (77.4\%). CD109 expression was associated with a higher histological tumor grade $(\mathrm{p}=.012)$. Twelve of 51 FIGO stage I tumors (23.5\%) and 51 of 69 FIGO stage II, III, and IV tumors $(73.9 \%)$ were positive for CD109 $(\mathrm{p}<.001)$. Thirteen of 45 tumors with $<10$ mitotic events $(28.9 \%)$ and 50 of 75 tumors with more than 10 mitotic events $(66.7 \%)$ were posi- tive for CD109 ( $<$.001). A high nuclear grade was not associated with CD109 expression (Table 4).

In the above results, CD109 positivity ratio varied according to the histologic type, and the following is the CD109 expression results in serous carcinoma that accounts for the largest percentage of OEC: Six of 13 FIGO stage I tumors (46.2\%) and 40 of 46 FIGO stage II, III, and IV tumors $(87.0 \%)$ were positive for CD109 ( $\mathrm{p}=.004)$. Histologic grade, high nuclear grade, and high mitotic counts were not associated with CD109 expression (Table 5).

\section{Association between CD109 expression and OEC prognosis}

CD109 expression was associated with overall survival ( $\mathrm{p}=$
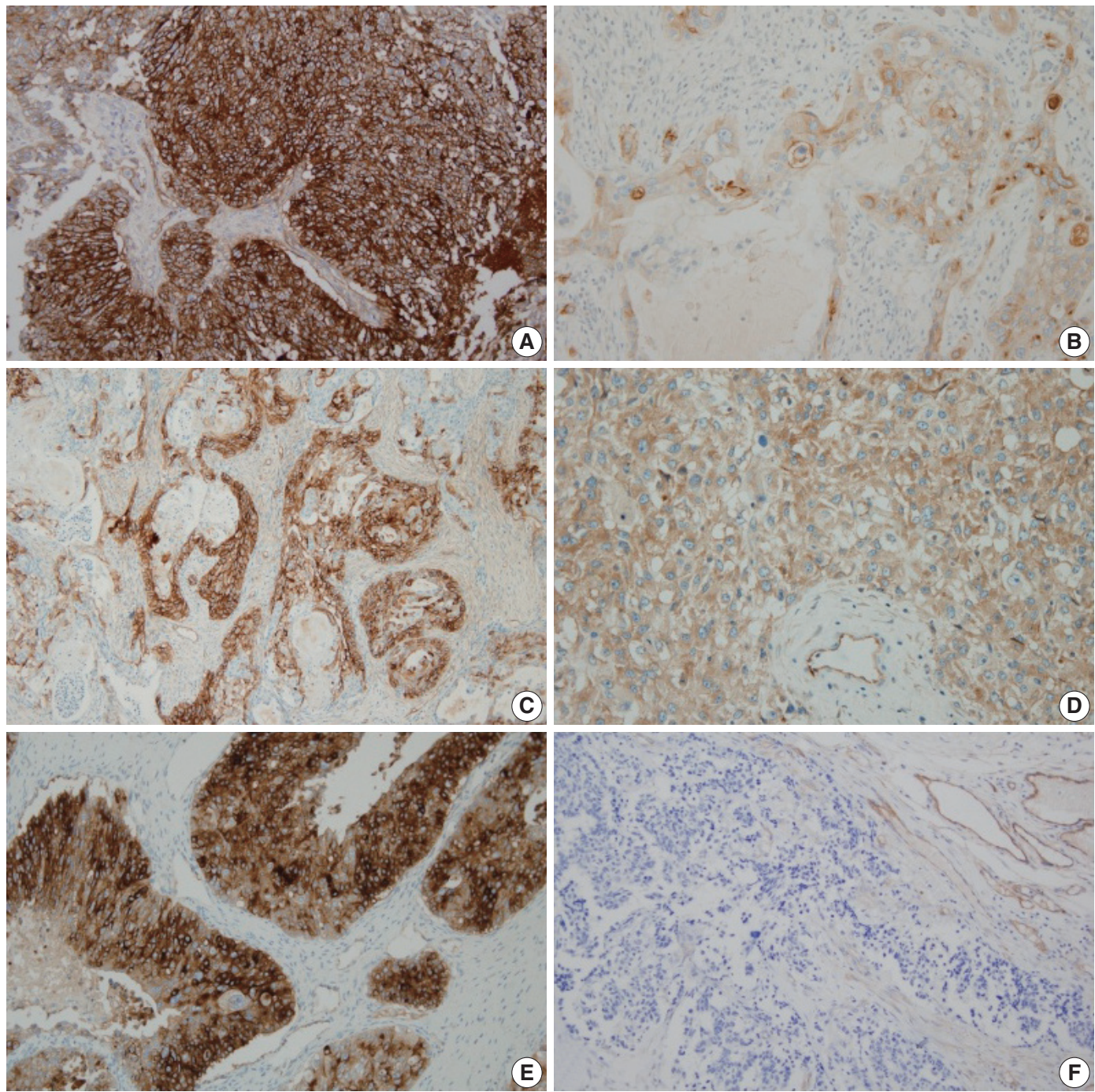

Fig. 1. Immunohistochemical staining of CD109 in ovarian epithelial carcinoma. (A) Positive in serous carcinoma. (B) Positive in mucinous carcinoma. (C) Positive in endometrioid carcinoma. (D) Positive in clear cell carcinoma. (E) Positive in undifferentiated carcinoma. (F) Negative in serous carcinoma. 
.020) (Fig. 2), but not recurrence-free survival ( $=$ = .290) (Fig. 3). The 5-year survival rate was $42.5 \%$ in patients with CD109-positive results and $64.8 \%$ in patients with CD109-negative results

Table 4. Correlation between CD109 expression and clinicopathological characteristics in ovarian epithelial carcinomas $(n=120)$

\begin{tabular}{lrrc}
\hline Parameter & Negative & Positive & p-value \\
\hline Histologic type & & & .000 \\
$\quad$ Serous & $13(22.0)$ & $46(78.0)$ & \\
Mucinous & $19(86.4)$ & $3(13.6)$ & \\
$\quad$ Endometrioid & $3(33.3)$ & $6(66.7)$ & \\
$\quad$ Clear cell & $22(81.5)$ & $5(18.5)$ & \\
$\quad$ Undifferentiated & $0(0)$ & $3(100)$ & \\
Histologic grade & & & .012 \\
$\quad$ Well differentiated & $19(67.9)$ & $9(32.1)$ & \\
$\quad$ Moderate differentiated & $29(47.5)$ & $32(52.5)$ & \\
$\quad$ Poorly differentiated & $9(29.0)$ & $22(71.0)$ & \\
Nuclear grade & & & .788 \\
I & $3(50.0)$ & $3(50.0)$ & \\
II & $29(44.6)$ & $36(55.4)$ & \\
III & $25(51.0)$ & $24(49.0)$ & \\
FIGO stage & & & .000 \\
I & $39(76.5)$ & $12(23.5)$ & \\
II, III and IV & $18(26.1)$ & $51(73.9)$ & \\
Mitoses (per 10HPFs) & & & .000 \\
1-9 & $32(71.1)$ & $13(28.9)$ & \\
$\geq 10$ & $25(33.3)$ & $50(66.7)$ & \\
\hline
\end{tabular}

Negative, less than $10 \%$ positive staining of tumor cells; Positive, more than $10 \%$ positive staining of tumor cells.

FIGO, Federation of Gynecology and Obstetrics; HPF, high power field. aPearson's chi-squared test.

Table 5. Correlation between CD109 expression and clinicopathological characteristics in serous carcinoma $(n=59)$

\begin{tabular}{lccc}
\hline Parameter & Negative & Positive & p-value \\
\hline Histologic grade & & & .351 \\
Well differentiated & $2(40.0)$ & $3(60.0)$ & \\
Moderate differentiated & $9(24.3)$ & $28(75.7)$ & \\
$\quad \begin{array}{l}\text { Poorly differentiated } \\
\text { Nuclear grade }\end{array}$ & $2(11.8)$ & $15(88.2)$ & \\
I & & & .850 \\
II & $0(0)$ & $1(100)$ & \\
III & $8(21.6)$ & $29(78.4)$ & \\
FIGO stage & $5(23.8)$ & $16(76.2)$ & \\
I & & & .004 \\
II, III and IV & $7(53.8)$ & $6(46.2)$ & \\
Mitoses (per 10HPFs) & $6(13.0)$ & $40(87.0)$ & \\
1-9 & & & .959 \\
10-19 & $3(25.0)$ & $9(75.0)$ & \\
$\geq 20$ & $5(21.7)$ & $18(78.3)$ & \\
\hline
\end{tabular}

Values are presented as number (\%).

FIGO, Federation of Gynecology and Obstetrics; HPF, high power field; Negative, less than $10 \%$ positive staining of tumor cells; Positive, more than $10 \%$ positive staining of tumor cells.

aPearson's chi-squared test. $(\mathrm{p}=.020)$ (Fig. 2). Analysis of the variables using multivariate analysis showed that $\mathrm{CD} 109$ expression was not an independent risk factor for overall survival due to its low reliability (hazard ratio [HR], 1.58; $\mathrm{p}=.160 ; 95 \%$ confidence interval [CI], 0.82 to 3.05 ) (Table 6). In addition, CD109 expression was not an only risk

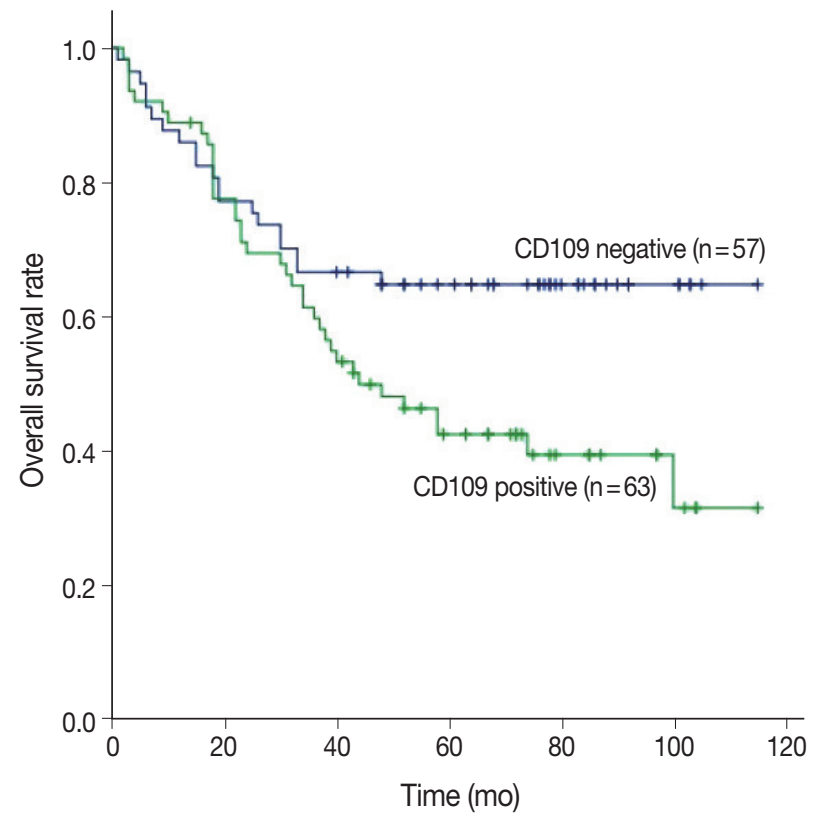

Fig. 2. Expression of CD109 protein is associated with overall survival. Prognosis is estimated by Kaplan-Meier plots $(p=.020)$.

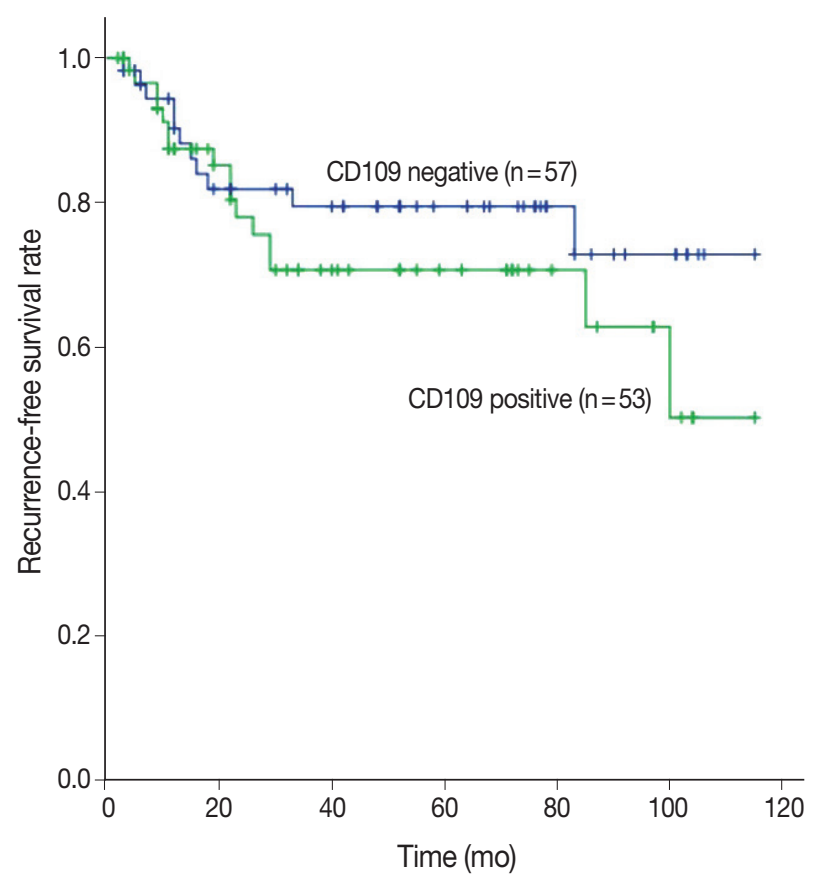

Fig. 3. Expression of CD109 protein is not significantly associated with recurrence. Prognosis is estimated by Kaplan-Meier plots ( $p=$ .290). 
Table 6. Multivariate analysis of overall survival $(n=120)$

\begin{tabular}{lccc}
\hline Variable & Hazard ratio & $\begin{array}{c}95 \% \text { confidence } \\
\text { interval }\end{array}$ & p-value \\
\hline $\begin{array}{l}\text { Histologic grade } \\
\text { Well differentiated }\end{array}$ & 1 & & \\
$\quad$ Moderate differentiated & 0.33 & $0.12-0.87$ & .020 \\
$\quad$ Poorly differentiated & 0.63 & $0.34-1.14$ & .130 \\
FIGO stage & & & \\
I & 1 & & \\
II, III, and IV & 0.17 & $0.07-0.38$ & $<.001$ \\
CD109 expression & & & \\
$\quad$ Negative & 0 & & \\
Positive & 1.58 & $0.82-3.05$ & .160 \\
\hline
\end{tabular}

Table 7. Multivariate analysis of recurrence-free survival $(n=120)$

\begin{tabular}{lccr}
\hline \multicolumn{1}{c}{ Variable } & Hazard ratio & 95\% confidence interval & $p$-value \\
\hline Histologic grade & & & \\
Well differentiated & 1 & & \\
Moderate differentiated & 0.34 & $0.09-1.26$ & .220 \\
Poorly differentiated & 0.53 & $0.21-1.32$ & .100 \\
FIGO stage & & & \\
I & 1 & & \\
II, III, and IV & 1.29 & $0.41-0.41$ & $<.001$ \\
CD109 expression & & & \\
$\quad$ Negative & 1 & & .110 \\
Positive & 2.06 & $0.83-5.09$ & \\
\hline
\end{tabular}

factor for recurrence-free survival (HR, 2.06; $\mathrm{p}=.110 ; 95 \% \mathrm{CI}$, 0.83 to 5.09 ) (Table 7). Therefore, CD109 expression is not an independent prognostic factor but is helpful for predicting the prognosis of patients with OEC.

Immunohistochemical staining was performed in 37 patients with serous carcinoma who received chemotherapy, 21 patients belonged to the good-response group and 16 patients belonged to the poor-response group. The poor-response group had higher rates of CD109 expression than the good-response group (93.8\% vs $66.7 \%, \mathrm{p}=.047$ ) (Table 8). Thus, CD109 is useful for predicting the chemotherapeutic effect in patients with OEC, especially serous carcinoma.

\section{CD109 mRNA levels in OECs}

All 12 fresh OEC samples were examined for CD109 expression by RT-qPCR. The samples were limited to serous carcinoma of OEC. CD109 mRNA was significantly upregulated in the poor-response group compared with the good-response group ( $\mathrm{p}=.001)$ (Fig. 4), suggesting that high CD109 expression level is associated with chemoresistance in patients with OEC.
Table 8. Chemotherapy response in correlation with CD109 expression

\begin{tabular}{lcc}
\hline \multirow{2}{*}{ Parameter } & \multicolumn{2}{c}{ CD109 expression } \\
\cline { 2 - 3 } & Negative & Positive \\
\hline Good response group & $7(33.3)$ & $14(66.7)$ \\
Poor response group & $1(12.5)$ & $15(93.8)$ \\
\hline
\end{tabular}

Values are presented as number (\%).

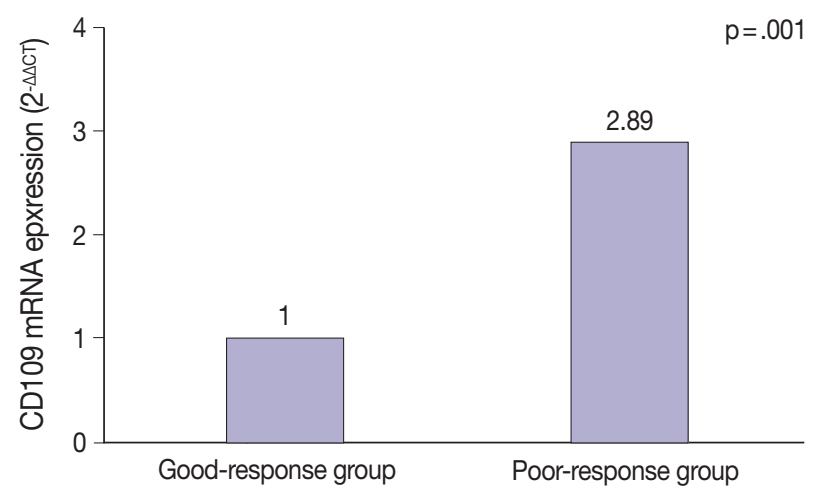

Fig. 4. Relationship between chemoresistance and CD109 mRNA expression $(n=12)$.

\section{DISCUSSION}

The physiological function of CD109 has not been studied, yet it is highly expressed in various types of malignancies and several normal tissues. ${ }^{8,20}$ It has recently been reported that CD109 expression is associated with tumor development and cell proliferation using human oral tumor tissues and cancer cell lines. ${ }^{21}$ In addition, $\mathrm{CD} 109$ can predict prognosis in tumors such as myxofibrosarcoma. ${ }^{15}$ In this study, we evaluated CD109 expression in OEC samples by immunohistochemical stains and RT-qPCR and found that an increased CD109 expression was associated with the prognosis and chemoresistance of OEC.

CD109 protein is a component of the TGF- $\beta 1$ receptor system. ${ }^{9}$ Tumor suppression occurs through the TGF- $\beta 1-$ signaling pathway in the early tumor stage; however, in malignantly transformed cells, TGF- $\beta 1$ is severely dysregulated, resulting in the loss of tumor suppression. ${ }^{9} \mathrm{CD} 109$ expression is followed by a complex formation with the type I TGF- $\beta 1$ receptor, which is required for the regulation of TGF- $\beta 1$ signaling in early tumor cells. ${ }^{22}$ Finally, CD109 degrades the TGF- $\beta 1$ receptor and blocks TGF- $\beta 1$ signaling, thereby preventing tumor suppression, ${ }^{23}$ which was confirmed in a mouse model. ${ }^{24}$ In CD109-deficient mice, the TGF- $\beta 1$ signaling pathway was enhanced and it suppressed skin tumorigenesis. $^{24}$

TGF- $\beta 1$ plays an important role in chemoresistance in breast cancer and squamous cell carcinoma. ${ }^{25,26}$ During TGF- $\beta 1$ expres- 
sion, cancer cells proliferate slowly in early tumor stage and lead to chemoresistance because they can be protected against DNAdamaging chemotherapeutic agents. ${ }^{26}$ In myxofibrosarcomas, tumors with high CD109 expression level showed decreased expression of TGF- $\beta 1$ with a good treatment effect. ${ }^{27}$ However, tumors with low CD109 expression and high TGF- $\beta 1$ expression showed chemoresistance. ${ }^{27}$ The current case and the other cases mentioned above indicate that there are other underlying mechanisms stronger than the CD109-TGF- $\beta 1$ signaling pathway. In our study, the relationship between CD109 expression and chemoresistance was identified, but TGF- $\beta 1$ expression and its role were not confirmed. If both CD109 and TGF- $\beta 1$ are found to be related to the chemoresistance of OEC, it will be helpful in treating patients. Therefore, further research is needed to confirm these findings in OEC.

CD109 expression has been associated with chemotherapeutic resistance in triple-negative breast cancers. ${ }^{28}$ After chemotherapy was administered to patients with CD109-positive triple negative breast cancer, $50 \%$ of the patients showed disease progression and none of them showed complete response..$^{28}$ The same result was reported when examining the CD109 expression and chemotherapeutic effect in breast cancer and glioblastoma. ${ }^{29,30}$ This may be due to the role of $\mathrm{CD} 109$ in the development of endothelial cells and induction of angiogenesis in contrary to the effect of chemotherapy regimen, which blocks tumor neovascularization. ${ }^{30}$ In this study, $\mathrm{CD} 109$ was expressed at a higher rate in the poor-response group than in the good-response group. In addition, the expression level of CD109 mRNA was 2.88-fold higher in the poor-response group than in the good-response group. However, unlike breast cancer, most OECs are diagnosed at advanced stages, making it difficult to divide the chemotherapy response group into more detailed subgroups.

$\mathrm{CD} 109$ is involved in the pathogenesis and prognosis of some tumors. CD109 overexpression is known to induce cell growth in oral squamous cell carcinomas, and CD109-positive oral dysplastic lesions develop into squamous cell carcinoma within 3 years. ${ }^{21}$ Although there is no correlation between the $\mathrm{CD} 109$ expression and mitosis, CD109 expression was associated with neoplastic cell growth. ${ }^{31}$ Recently, a study suggested that CD109 expression is managed by cancer stem-like cells/cancer-initiating cells, which can initiate tumorigenesis and tumor growth in sarcomas. ${ }^{14}$ Therefore, in the presence of cancer stem cells, CD109 expression may increase and malignancies or recurrence/metastasis may develop. In our study, CD109 expression was higher in the advanced stage, but OEC precursors such as borderline ovarian tumor is not included in the study. More research that can determine the association of CD109 with neoplastic cell growth in ovary tissue may be helpful in early detection and treatment of patients.

Based on our review, this is the first study on the relationship between CD109 expression and prognosis of OEC. The present study demonstrated that CD109 is highly expressed in OEC tissues. Immunohistochemical analysis of CD109 expression is useful for predicting overall survival but not recurrence-free survival. Regarding the chemotherapeutic effect on serous carcinoma, the poor-response group expressed increased mRNA levels of CD109 as compared to the good-response group. Therefore, identification of CD109 expression may help in predicting the survival and chemotherapeutic effect in patients with serous carcinoma of OEC. Further analysis of other factors related to $\mathrm{CD} 109$ is needed for careful management of patients with serous carcinoma of OEC.

\section{ORCID}

So Young Kim: https://orcid.org/0000-0003-3076-9147

Kyung Un Choi: https://orcid.org/0000-0002-3848-1781

Chungsu Hwang: https://orcid.org/0000-0001-9520-0301

Hyung Jung Lee: https://orcid.org/0000-0002-2995-6060

Jung Hee Lee: https://orcid.org/0000-0003-3003-2217

Dong Hoon Shin: https://orcid.org/0000-0002-4980-9295

Jee Yeon Kim: https://orcid.org/0000-0002-0503-984X

Mee Young Sol: https://orcid.org/0000-0002-2655-6395

Jae Ho Kim: https://orcid.org/0000-0003-4323-4790

Ki Hyung Kim: https://orcid.org/0000-0003-2364-5875

Dong Soo Suh: https://orcid.org/0000-0001-5785-4355

Byung Su Kwon: https://orcid.org/0000-0002-9586-0200

\section{Author Contributions}

Conceptualization: SYK, KUC.

Data curation: $\mathrm{CH}$, KHK, DSS, BSK.

Formal analysis: SYK, CH, HJL.

Funding acquisition: KUC, JHK.

Investigation: SYK, KUC, DHS, JYK .

Methodology: SYK, JHL, DHS, JYK, KUC.

Project administration: SYK, KUC, JHK.

Resources: KUC, KHK, DSS, BSK.

Supervision: KUC, JHK.

Validation: DSS, JYK.

Writing—original draft: SYK, KUC.

Writing—review \& editing: HJL, JHL, DHS, JHK, MYS. 


\section{Conflicts of Interest}

The authors declare that they have no potential conflicts of interest.

\section{Acknowledgments}

This work was supported by the National Research Foundation of Korea (NRF) grant funded by the Korea government (MSIT) (NRF-2017R1A2B4009021) and the Korea Health Technology R\&D Project, Ministry of Health and Welfare (HI17 C1635). The biospecimens for this study were provided by the Biobank of Pusan National University Hospital, a member of the National Biobank of Korea, which is supported by the Ministry of Health, Welfare and Family Affair.

\section{REFERENCES}

1. Montes AF, Gomez JG, Viejo MN, Bermejo MA, Urrutia SA, Mata JG. Epidemiology and etiology of ovarian cancer. In: Farghaly S, ed. Ovarian cancer: basic science perspective. Vancouver: InTech, 2012; 1-16.

2. Flam F, Einhorn N, Sjövall K. Symptomatology of ovarian cancer. Eur J Obstet Gynecol Reprod Biol 1988; 27: 53-7.

3. Goff BA, Mandel L, Muntz HG, Melancon CH. Ovarian carcinoma diagnosis. Cancer 2000; 89: 2068-75.

4. Liu J, Matulonis UA. New strategies in ovarian cancer: translating the molecular complexity of ovarian cancer into treatment advances. Clin Cancer Res 2014; 20: 5150-6.

5. Hartmann LC, Podratz KC, Keeney GL, et al. Prognostic significance of p53 immunostaining in epithelial ovarian cancer. J Clin Oncol 1994; 12: 64-9.

6. Luo H, Xu X, Ye M, Sheng B, Zhu X. The prognostic value of HER2 in ovarian cancer: a meta-analysis of observational studies. PLoS One 2018; 13: e0191972.

7. Lin M, Sutherland DR, Horsfall W, et al. Cell surface antigen CD109 is a novel member of the alpha(2) macroglobulin/C3, C4, C5 family of thioester-containing proteins. Blood 2002; 99: 1683-91.

8. Hasegawa M, Hagiwara S, Sato T, et al. CD109, a new marker for myoepithelial cells of mammary, salivary, and lacrimal glands and prostate basal cells. Pathol Int 2007; 57: 245-50.

9. Leivonen SK, Kähäri VM. Transforming growth factor-beta signaling in cancer invasion and metastasis. Int J Cancer 2007; 121: 2119-24.

10. Zhang JM, Hashimoto M, Kawai K, et al. CD109 expression in squamous cell carcinoma of the uterine cervix. Pathol Int 2005; 55: 165-9.

11. Sato T, Murakumo Y, Hagiwara S, et al. High-level expression of CD109 is frequently detected in lung squamous cell carcinomas.
Pathol Int 2007; 57: 719-24.

12. Dong F, Lu C, Chen X, Guo Y, Liu J. CD109 is a novel marker for squamous cell/adenosquamous carcinomas of the gallbladder. Diagn Pathol 2015; 10: 137.

13. Ozbay PÖ, Ekinci T, Yiğit S, et al. Investigation of prognostic significance of CD109 expression in women with vulvar squamous cell carcinoma. Onco Targets Ther 2013; 6: 621-7.

14. Emori M, Tsukahara T, Murase M, et al. High expression of CD109 antigen regulates the phenotype of cancer stem-like cells/cancerinitiating cells in the novel epithelioid sarcoma cell line ESX and is related to poor prognosis of soft tissue sarcoma. PLoS One 2013; 8: e84187.

15. Emori M, Tsukahara T, Murata K, et al. Prognostic impact of CD109 expression in myxofibrosarcoma. J Surg Oncol 2015; 111: 975-9.

16. Kurman RJ, Carcangiu ML, Herrington CS, Young RH. WHO classification of tumours of female reproductive organs. 4th ed. Lyon: International Agency for Research on Cancer, 2014; 11-40.

17. Shimizu Y, Kamoi S, Amada S, Akiyama F, Silverberg SG. Toward the development of a universal grading system for ovarian epithelial carcinoma: testing of a proposed system in a series of 461 patients with uniform treatment and follow-up. Cancer 1998; 82: 893-901.

18. Pecorelli S, Benedet JL, Creasman WT, Shepherd JH. FIGO staging of gynecologic cancer. 1994-1997 FIGO Committee on Gynecologic Oncology. International Federation of Gynecology and Obstetrics. Int J Gynaecol Obstet 1999; 65: 243-9.

19. Yuan JS, Reed A, Chen F, Stewart CN Jr. Statistical analysis of realtime PCR data. BMC Bioinformatics 2006; 7: 85.

20. Hashimoto M, Ichihara M, Watanabe T, et al. Expression of CD109 in human cancer. Oncogene 2004; 23: 3716-20.

21. Hagiwara S, Murakumo Y, Sato T, et al. Up-regulation of CD109 expression is associated with carcinogenesis of the squamous epithelium of the oral cavity. Cancer Sci 2008; 99: 1916-23.

22. Hagiwara S, Murakumo Y, Mii S, et al. Processing of CD109 by furin and its role in the regulation of TGF-beta signaling. Oncogene 2010; 29: 2181-91.

23. Cuppini L, Calleri A, Bruzzone MG, et al. Prognostic value of CD109+ circulating endothelial cells in recurrent glioblastomas treated with bevacizumab and irinotecan. PLoS One 2013; 8: e74345.

24. Hasegawa M, Moritani S, Murakumo Y, et al. CD109 expression in basal-like breast carcinoma. Pathol Int 2008; 58: 288-94.

25. Tao J, Li H, Li Q, Yang Y. CD109 is a potential target for triple-negative breast cancer. Tumour Biol 2014; 35: 12083-90.

26. Mancuso P, Calleri A, Gregato G, et al. A subpopulation of circulating endothelial cells express CD109 and is enriched in the blood of cancer patients. PLoS One 2014; 9: e114713.

27. Tsai YL, Ha DP, Zhao H, et al. Endoplasmic reticulum stress acti- 
vates SRC, relocating chaperones to the cell surface where GRP78/ CD109 blocks TGF-beta signaling. Proc Natl Acad Sci U S A 2018; 115: E4245-54.

28. Sunagawa M, Mii S, Enomoto A, et al. Suppression of skin tumorigenesis in CD109-deficient mice. Oncotarget 2016; 7: 82836-50.

29. Bhola NE, Balko JM, Dugger TC, et al. TGF-beta inhibition enhances chemotherapy action against triple-negative breast cancer. J Clin In- vest 2013; 123: 1348-58.

30. Oshimori N, Oristian D, Fuchs E. TGF-beta promotes heterogeneity and drug resistance in squamous cell carcinoma. Cell 2015; 160: 963-76.

31. De Vita A, Recine F, Mercatali L, et al. Myxofibrosarcoma primary cultures: molecular and pharmacological profile. Ther Adv Med Oncol 2017; 9: 755-67. 